\title{
Circuit
}

Musiques contemporaines

\section{Les usagers de la musique}

L'écoute des amateurs

\section{Users of Music}

\section{The Listening of Music Lovers}

\section{Antoine Hennion}

Volume 14, numéro 1, 2003

Qui écoute? 2

URI : https://id.erudit.org/iderudit/902298ar

DOI : https://doi.org/10.7202/902298ar

Aller au sommaire du numéro

Éditeur(s)

Les Presses de l'Université de Montréal

ISSN

1183-1693 (imprimé)

1488-9692 (numérique)

Découvrir la revue

Citer cet article

Hennion, A. (2003). Les usagers de la musique : l'écoute des amateurs. Circuit, 14(1), 19-32. https://doi.org/10.7202/902298ar

\section{Résumé de l'article}

Cette contribution est écrite à partir d'entretiens, d'observations ou d'expériences réalisés en équipe ces dernières années auprès d'amateurs ayant des attachements de nature et d'intensité diverses à la musique. Il s'agit de se mettre à l'écoute de leur écoute, en essayant le plus possible de laisser leur espace propre aux formes très variées qu'elle peut prendre. Loin d'être inférieures, incompétentes, passives ou obsédées par le seul plaisir immédiat du son ou de l'image facile, les oreilles des amateurs sont actives, créatives. Elles jouent avec les nouveaux moyens de la musique, la disponibilité démultipliée d'un répertoire, la manipulation instantanée de musiques diverses. Elles travaillent les positions de l'écoute, à commencer par la disposition physique de soi. Elles débouchent souvent sur un art de faire très personnel, inventant de véritables façons de se mettre en musique. Mettre l'accent sur l'écoute, c'est donc réintroduire l'hétérogénéité irréductible d'un réel-événement, fait de plis et de tissages. Non pas une oeuvre et un auditeur : des corps, des dispositifs et des dispositions, de la durée, un objet insaisissable, un instant qui passe, des états qui surgissent. Après tout, hors des laboratoires et des écoles, qu'est d'autre la musique?
Ce document est protégé par la loi sur le droit d'auteur. L’utilisation des services d’Érudit (y compris la reproduction) est assujettie à sa politique d'utilisation que vous pouvez consulter en ligne.

https://apropos.erudit.org/fr/usagers/politique-dutilisation/ 


\title{
Les usagers de la musique L'écoute des amateurs
}

\author{
Antoine Hennion
}

Le thème de l'écoute n'est vraiment pas taillé sur mesure pour le sociologue - c'est sans doute ce qui en fait l'intérêt. Dans cette courte contribution, écrite à partir des entretiens, des observations ou des expériences que j'ai réalisés avec d'autres chercheurs et étudiants ces dernières années ${ }^{\prime}$, je vais essayer de faire parler des amateurs, en relisant librement certains passages pour nous mettre en quelque sorte à l'écoute de leur écoute. J'y vois un avantage, celui d'éviter la définition normative, explicite ou plus insidieuse, qu'entraîne avec elle toute tentative de théorisation de l'écoute : dire ce qu'elle est, c'est vite dire ce qu'elle doit être. En écoutant des amateurs, on voit au contraire se déployer une variété imprévue d'écoutes. La formule comporte aussi un danger - le nommer aidera peutêtre à le contenir - celui de prendre pour argent comptant les formulations des amateurs. II ne s'agit pas de les suspecter de mentir, mais de prendre la mesure du fait que l'écoute en question, telle que nous allons tenter de la saisir, ne se met pas toute seule en mots, loin de là. Outre que la verbalisation obtenue modifie le rapport de l'interviewé à ce dont il parle au fur et à mesure qu'il s'exprime, elle se fait en réaction à la situation d'entretien : jeu d'opposition et de séduction par rapport à l'intervieweur, anticipations d'attentes réelles ou supposées, etc. Sans prétendre régler cet aspect des choses, j'essaierai le plus possible de donner des éléments qui situent les entretiens, lorsque cela semble pertinent, et d'expliciter les interactions que j'ai pu saisir.

À première vue, les écoutes ainsi mises en scène feront peutêtre penser aux stéréotypes amusants dégagés par Adorno (Adorno, 1994) dans sa célèbre typologie des auditeurs allant, de haut en bas, de l'écoute structurelle, la seule "autorisée», aux écoutes anecdotiques et sentimentales, en passant par celle des bons élèves qui font des efforts. Mais, si on laisse "sonner" chacune d'elles, si on leur laisse leur espace propre, nous verrons qu'en réalité, elles nous emmènent aux antipodes de l'esprit normatif de cette typologie, qui n'est que le décalque imaginaire du point de vue du compositeur. Loin d'être inférieures, incompétentes, passives ou obsédées par le seul plaisir immédiat du son ou de l'imaginaire facile, les oreilles des amateurs sont actives, créatives. Elles jouent avec les nouveaux moyens de la musique,

1. Voir HENNION et MAISONNEUVE (2000), HENNION (2002), MAISONNEUVE, TEIL et HENNION (2002), TEll et HENNION (2003), pour des comptes rendus de divers aspects de ces enquêtes réalisées dans de multiples directions, sur les amateurs et, plus généralement, les formes d'attachement des personnes aux objets de leurs pratiques (musique, vin, collections, etc.). Un livre de synthèse sur « les protocoles du goût», écrit avec G. Teil, doit paraître en 2004 aux éditions Métailié. 
la disponibilité démultipliée d'un répertoire, la manipulation instantanée de musiques diverses, l'invention de positions d'écoute, à commencer par la disposition physique de soi, et débouchent souvent sur un art de faire très personnel, inventant de véritables façons de se mettre en musique.

\section{Des écoutes en acte}

Cette expérimentation personnelle, idiosyncrasique, de l'écoute en situation, c'est aussi à la sociologie du goût qu'elle vient lancer un défi. Par une inversion critique caractéristique, hautement contestable, celle-ci tend en effet à ne faire de l'expérience ordinaire de l'écoute qu'une application inconsciente des cadres officiels du goût - pour rapporter ensuite ceux-ci à leurs déterminations sociales. C'est là renverser l'ordre des choses, faire du goût une propriété bien déterminée, aux deux sens du terme : à la fois passive et fixée. Mais c'est ce jugement stable, cohérent avec les facteurs qui le déterminent, qui est rare, et ne se formule que dans des conditions très particulières, où précisément le goût est d'abord affirmation d'une identité et réponse à un jugement social - par exemple, devant un sociologue ou dans un questionnaire ${ }^{2}$. En face d'un objet inconnu, on est bien loin de retrouver cette belle cohérence entre soi-même et ses propres sensations... Ce n'est pas ce goût tout fait, la cible de nos analyses : c'est l'acte de goûter, les gestes qui le permettent, les savoirfaire qui l'accompagnent, les petits ajustements en continu qui l'aménagent et favorisent sa félicité et sa reproduction - comme le fera l'effort même pour l'exprimer devant moi. Autre façon de présenter ce texte, donc : il vise à produire une mise en mots de l'écoute en acte, en situation, avec ses trucs et ses bricolages, loin de tout espace de justification publique, mais attentive à son propre succès - ce caractère réflexif de l'écoute au moment où elle se déroule favorisant ensuite sa reprise verbalisée dans la relation à un intervieweur ou à un dispositif expérimental.

Je mets donc tout de suite de côté l'écoute scolaire, ou "obligée", comme on dit au clavecin, i'entends par là celle que préconisent les compositeurs eux-mêmes et leurs agents, promoteurs de la fameuse écoute structurelle. Je l'exclus d'entrée, non qu'elle n'ait pas lieu d'être, mais parce qu'elle n'a pas d'épaisseur propre, qu'elle n'est qu'un négatif de l'esthétique prônée par l'œuvre. Si on libère l'écoute de cette servilité noblement acceptée, le thème a en effet le mérite de réintroduire une grande incertitude dans la musique, tant du côté de l'esthétique, comme l'a montré le premier numéro consacré à ce thème, que du côté du sociologue, intéressé par ce qu'on fait de la musique et par ce qu'elle fait, ou du musicologue, qu'elle oblige à quitter des yeux les seules partitions.

l'écoute ainsi conçue est d'abord un décentrement. Elle ne se réduit pas si facilement ni à l'œuvre, bardée de ses prescriptions et de ses modes d'emploi, ni à
2. À la fois sans cesse interrogé de façon réflexive, mais utilisé de façon exclusive, c'est le mode d'interprétation des goûts mis en œuvre par P. Bourdieu tout au long de la Distinction (1979), il est vrai à partir d'un questionnaire particulièrement réducteur dans le cas de la musique. 
l'auditeur, bardé de ses goûts et de ses déterminations. C'est cette indiscipline qui la rend suggestive. Elle est passage, et non objet. Souligner ainsi son flottement, comme disent ailleurs les psychanalystes, c'est marquer un temps d'arrêt sur quelque chose qui arrive (ou non), au lieu de rapporter précipitamment l'événement musical à du connu ou, ce qui est peutêtre pis, à du «à connaître» : à l'œuvre elle-même, au langage et aux formes musicales, d'un côté ; aux compétences cognitives ou psychologiques d'un auditeur, de l'autre. Mettre l'accent sur l'écoute, c'est réintroduire l'hétérogénéité irréductible d'un réel-événement, fait de plis et de tissages, qu'une longue tradition nourrie de la dualité objet-sujet nous a appris à ne pas trop aimer. Non pas une œuvre et un auditeur : des corps, des dispositifs et des dispositions, de la durée, un objet insaisissable, un instant qui passe, des états qui surgissent...

Après tout, hors des laboratoires et des écoles, qu'est d'autre la musique?

\section{Une autre histoire de la musique}

Car l'écoute n'est pas qu'un instant, elle est aussi une histoire. C'est toute la musique que le mot remet en scène sous un autre jour, non plus, cette fois, dans le présent d'un contact à des sons qui passent, mais dans la durée improbable d'une lente invention, celle d'un art et d'une technique de l'écoute pour l'écoute. Production d'espaces et de durées propres, de scènes et de dispositifs "dédiés", comme on dit en informatique, constitution progressive et évolutive d'un répertoire, entraînement des corps et des esprits, formation d'un milieu de professionnels et d'un cercle d'amateurs, c'est l'autre versant de l'écoute : la musique comme délégation du pouvoir de nous émouvoir à un ensemble d'œuvres, devenu cible d'une écoute privilégiée.

D'un point de vue disciplinaire, cet aspect historique de l'écoute est tout aussi dérangeant, par sa fausse évidence, que ne l'est l'hétérodoxie têtue de l'écoute instantanée : le fait même d'écouter la musique est une position étrange, dont on a du mal à percevoir le caractère paradoxal une fois qu'on l'occupe et qu'elle nous est devenue si naturelle ${ }^{3}$ (Weber, 1997). Se placer devant un objet identifié, qui "doit» être écouté, dont on a, pour cet objectif, équipé la perception de techniques, de mots, de toutes les prothèses nécessaires, et qui est capable de répondre à cette attente, c'est à la fois, comme le souligne joliment Szendy, ce qui est le plus fondamental, le geste qui fait la musique telle que nous l'entendons, et ce qui est la part la moins visible de nos opérations musicales. Du moins lorsqu'on tourne autour des musiques savantes : il suffit de s'éloigner de ses cercles étroits, et de dériver soit vers les musiques dites actuelles, soit au contraire vers les musiques ethniques et populaires, pour vite retrouver la multiplicité hétérogène de relations mêlées, d'«événements", au sens des producteurs de spectacle, dans lesquels on serait bien en peine de faire le départ entre le plaisir d'un collectif, les sensations des corps, les formats d'un moment organisé, et les éléments musicaux d'une performance ${ }^{4}$.
3. L'historien social de la musique et du concert $W$. Weber posait crûment la question, se demandant tout simplement si on écoutait la musique au XVIII' siècle (1997). Au-delà de la variété connue des attitudes durant les spectacles, le problème est celui de l'anachronisme naturalisant qu'il y a à utiliser les mêmes mots lécoute, musique, œuvre...) pour décrire des situations historiquement aussi opposées que la musique de cour, le concert moderne et le disque. Comme le dit fort bien P. Szendy (2001), les oreilles ont une histoire!

4. Au point qu'on peut se demander, en poussant peut-être le raisonnement à sa limite, si aujourd'hui, sur les scènes de la techno, la surpuissance des équipements sonores n'est pas d'abord un moyen d'empêcher l'écoute, pour refaire passer la musique du côté des techniques sociales du transport et de la fusion collective en l'arrachant à l'attention sélective inventée par les dispositifs classiques. 


\section{À l'écoute de l'écoute des amateurs}

Loin de la création, à l'autre extrémité de la chaîne musicale, c'est donc à l'écoute de quelques amateurs que je vais m'intéresser, comme ensemble de techniques propres, de moyens d'appropriation, de dispositifs d'attention, d'usages inventés sur le tas ou minutieusement élaborés, permettant d'entrer en contact avec de la musique. Sélection aléatoire ou programmée, écoute flottante ou sur-ciblée, modulation du volume, répétition à volonté, retours arrière, classements et regroupements, insertion dans les activités les plus inattendues ${ }^{5}$ : étroitement liée à l'équipement discographique et à ses développements numériques, il existe désormais une véritable "fonction-écoute», qui se décline sous de multiples modalités.

Les exemples qui suivent visent surtout à en suggérer la diversité - mais peutêtre aussi, au passage, à mettre en valeur l'inventivité de ces amateurs-usagers, qui font penser aux promeneurs de Michel de Certeau ou aux cuisiniers du dimanche de Luce Giard (1980).
5. De l'aérobic aux parties-souvenirs, voir les exemples amusants donnés par Tia DeNora (2000).

\section{Benoît : où me placer pour entendre la musique?}

Je me suis formé à la musique par le chant choral. C'est une banalité, pour bien des jeunes qui sont passés par là, mais je dis ça en un sens très technique, si tu veux, je ne sais pas si c'est un truc à moi, mais c'est là que j'ai appris à entendre la musique, en suivant exprès une voix du milieu. Et je fais toujours ça maintenant, moi, la musique, je l'entends du milieu! Je t'explique, moi je n'ai jamais fait de solfège, i'étais perdu... tant que j'ai juste suivi les autres ténors, un peu en retard, tout en étant écrasé par la voix principale, au soprano, je n'ai rien compris. Et puis un beau jour, je ne sais pas, pour m'amuser ou parce qu'elle sonnait bien, i'ai suivi la voix des altos, tout en chantant la mienne, le ténor, et $j$ 'en suis resté estomaqué. J'entendais tout, comme dans un espace à plusieurs dimensions, moitié hors de moi, moitié en moi, ma stéréo à moi!

Après j'ai développé le truc, j'apprenais ces voix intermédiaires et je les recherchais ensuite sur les disques, c'était une joie intense. Maintenant je fais ça moins souvent, mais je me souviens d'avoir été absolument émerveillé par ces disques, enfin par cette façon de me passer des morceaux comme ça, qu'on avait chantés, en "partant " d'une voix, la mienne ou mieux une autre, mais une du milieu, ou la basse des fois, mais je n'entends pas aussi bien les basses. Je me souviens de grands chœurs à la Haendel, d'un motet de Bach, mais même des œuvres moins connues, et toutes ces polyphonies de la Renaissance, bien sûr.

Oui, c'est ça qui m'a fait aimer la musique. Et supporter la chorale! 
Avant tout recours au disque, Benoît s'est servi du médium à sa disposition, la grosse chorale de son école catholique de province, pour se fabriquer une "prise" vers la musique 6 . Tout est déjà dans ce "truc à lui » : l'appui sur les autres, la redéfinition de son oreille et l'entraînement musical que cela lui a donné, le "retour » de l'objet lui-même, en effet plus prêt à se laisser domestiquer et à révéler ses richesses à Benoît ainsi que par une écoute globale, indifférenciée, "écrasée" par le soprano, comme il le dit très justement. Et puis, bien sûr, la jubilation autovalorisante de cette découverte, et enfin l'accès rendu possible au monde "normal» de la musique classique, celui des disques et de la radio, demeuré jusque-là hors de portée de Benoît, même lorsqu'il chantait.
6. Sur cette notion de prise, très riche, et les développements qu'on peut en tirer pour améliorer la capacité de la sociologie du goût à prendre en considération les objets sur lesquels il porte, voir BESSY et CHATEAURAYNAUD (1995). Fautil préciser que l'interviewé ne s'engage dans une telle description que s'il pense qu'elle a un sens pour son interlocuteur? Celui-ci doit donc trouver des moyens légers de lui signaler à la fois son intérêt pour la pratique en question, et un minimum de connaissance de l'univers des chorales.

\section{Dora : le divan de l'écoute}

Très souvent, i'écoute allongée, ça me fait un bien fou, j'ai une bonne chaîne maintenant, ce que je n'avais jamais eu, et que j'avais toujours un peu snobé, tu vois le genre, "la musique c'est pas la Hifi", etc., en fait c'est con, le son lui-même fait énormément, mais justement pas sur le rapport intello à la musique, la partie œuvre, au contraire sur la partie physique, corporelle, c'est hyperphysique, le son, ça vibre, on rentre en résonance, on entend la musique autrement en se laissant faire par le son.

J'éteins en général, je fais ça plus quand mon mari n'est pas là, par exemple que je rentre tard et que je suis crevée, j'ai un truc à plusieurs CD, je prends mes piles et j'en choisis comme ça, à l'impulse, i'en ôte un, i'en reprends un - j'achète bien plus qu'avant, un peu au hasard, enfin pas au hasard mais très ouvert, i'aime un peu tout - et quand j'ai mis mes six disques, je m'allonge avec la télécommande et j'écoute, je saute la plage quand je veux changer, ou que je cherche un air que j'aime, c'est le bonheur absolu... Des fois c'est de la détente, ou je m'endors carrément, mais en général non, i'écoute au contraire vraiment, beaucoup mieux qu'en concert, c'est ça que permet le grand son, par rapport à un appareil lambda où tu as juste la musique elle-même, la "parto" qui passe, quoi, où c'est très bien d'un point de vue musical, l'œuvre... je ne sais pas comment dire, mais c'est plat pour le son, ça passe devant toi... tandis que sur mon divan comme ça, ça se déroule en toi, tu es dedans, c'est incroyable comme ça prend.

Moi ça me bouleverse, je suis très sensible, émotive, nerveuse, c'est presque une drogue, ou au contraire une thérapie, je ne sais pas... J'aime beaucoup aller aux concerts, aussi, mais ça n'a rien à voir, c'est pour voir les musiciens, les chanteurs surtout, je m'identifie à eux, je les vois faire la musique, c'est extra aussi mais ce n'est plus de l'écoute, c'est le concert, quoi, on est avec eux. Alors qu'au contraire, on est tout seul avec la musique, en écoutant comme ça.

$C^{\prime}$ est ici, remarquablement analytique /pour une amatrice qui reprend par ailleurs sans cesse le flambeau de l'anti-intellectualisme de la musique, en particulier 
en face d'un sociologue, pour insister en souriant sur l'intimité et la sensualité de son rapport à la musiquel, un véritable mode d'emploi de l'écoute "HiFi »-isée en tant qu'elle se distingue radicalement de l'écoute vivante. C'est d'autant plus intéressant que, sans se poser la question de leur relation, Dora aime bien aussi le concert, et qu'il n'y a donc dans sa description aucun plaidoyer plus général, défendant la musique "en boîte" contre la musique "vivante», etc. : non, juste le récit d'une façon de démultiplier les dispositions de corps et d'esprit les plus favorables aux effets, d'abord sensoriels, mais aussi psychologiques et imaginaires, et pourquoi pas musicaux, d'œuvres enfin "sous la main" - ce qui est en effet un élément clé de la "discomorphose" de la musique ${ }^{7}$.

De la disponibilité d'un répertoire à sa facilité d'acquisition et de sélection sur le moment, de l'importance de la posture physique à celle de la "chaîne " de l'audition qui va du corps au son lui-même qui l'envahit, Dora prend au sérieux l'écoute comme un état qui doit s'installer, dans son lieu et son temps, puis se "laisser faire", un peu comme une séance de relaxation solitaire mâtinée d'un rappel d'ambiances au contraire collectives (elle me parlera plus loin de l'acoustique d'église qu'elle obtient avec son installation, pour les chœurs ou les gros ensembles orchestraux). Tous les facteurs de cette inversion du rapport entre le disque comme enregistrement et le disque comme producteur de musique sont mis en place, et méticuleusement exploités.

Comment mieux sentir que le disque, en en faisant quelque chose à écouter, a créé une nouvelle musique ? Jusque-là, comme elle le dit spontanément à propos du concert, la musique était d'abord quelque chose à faire ly compris pour ses spectateursl, et le plus souvent à faire ensemble. Car cette première opposition, entre un objet et une relation, est ici redoublée de façon très forte par un autre contraste, bien dans le sillage de la révolution discographique, entre la solitude recherchée de ses séances d'écoute et le caractère social de la performance publique.

\section{Ahmed et le TGV : la musique mise en mouvement}

Je me suis acheté un baladeur pour $C D$, d'assez bonne qualité, et un étui pour transporter $24 C D$, et pendant les trois ans où j'ai fait Paris-Lyon Lyon-Paris deux fois par semaine, une fois que j'ai accepté une fois pour toutes que je n'arriverais pas à travailler dans le TGV après des cours, j'ai commencé à écouter de la musique systématiquement, d'abord des choses que je connaissais, de la musique dansante, brésilienne, arabo-andalouse, des pots-pourris, puis de plus en plus des choses que je ne connaissais pas. Je demandais à Annie, de plus en plus de classique, et hop, et alors pour moi c'est définitivement associé au TGV, je suivais le paysage en associant à la musique. Pas à telle musique, à la musique en général, c'est quelque chose qui passe, le fait de ne plus entendre autour de moi, mais de voir défiler un paysage en entendant défiler 
la bande, quoi, je lisais les rythmes dans les vallons, les changements de lumière... je dis ça de façon poétique mais c'est plus simple que ça, en vrai, je suis architecte aussi, c'est peutêtre que j'avais trouvé enfin le truc pour «lire » la musique dans un espace et la comprendre, c'est ça, je m'étais fait mon écran à moi.

Je ne vais plus à Lyon, quand je reprends le train je n'y pense pas toujours, ou je n'ai pas mon baladeur, et puis de toutes façons ce n'est plus ça, il fallait le côté régulier, je crois... Côté musiques, ça a fait un truc bizarre, c'est que je connais plein de musiques, surtout quand je reprends des disques d'Annie, mais je ne sais absolument pas ce que c'est, je ne retenais pas les pochettes, et je n'y connaissais vraiment rien, donc moi, même ce qu'elle croit évident, baroque ou ancien, musique de chambre, opéra, moi je n'en sais rien, je les connais par cœur mais je ne sais pas si c'est du Mozart ou du moderne!

Cette fois, l'accroche, moins orthodoxe encore, n'en a pas moins "ouvert» à Ahmed tout un espace de musiques jusque-là totalement ignorées, et que sur le plan verbal, par une sorte de fidélité ironique à son passé (il est à la fois fils d'immigrés algériens et un professionnel reconnu, comme il le signale au passage dans l'entretien), il continuera à laisser délibérément dans un anonymat original, contrastant avec la précision de ses souvenirs de voyageur - dans cette façon d'apprivoiser un patrimoine tout en le maintenant à distance (en somme, il lui refuse le baptême), il y a probablement une part de défi, difficile à mesurer avec précision, en particulier vis-à-vis du sociologue, liée à sa double identité sociale, à laquelle il peut supposer que son vis-à-vis est particulièrement attentif.

Ahmed s'est inventé une sorte de lanterne magique musicale. L'expression très personnelle avec laquelle il livre son expérience, la dépendance assumée aux goûts de sa compagne, à qui il délègue entièrement son approvisionnement, et le côté inhabituel $d$ 'un attachement au classique obtenu par ce moyen, ne font que mieux mettre en valeur le mode de fonctionnement comme écran "interne", comme il dit bien, que l'écoute au baladeur favorise, sous des formats sans doute variés.

\section{Philippe : la discothèque comme harem imaginaire}

L'un des tout premiers amateurs que j'ai interrogés, au début de l'enquête, m'avait alors livré une caricature du bon entretien pour étudiant en DEA de socio : ses origines familiales, sa sœur qui faisait du violon, l'oncle et la tante qui l'emmenaient petit au concert, la première fois qu'il est allé à l'opéra (une expérience inoubliable), son métier actuel (médecin), et ses goûts : le grand opéra et la musique de chambre.

Par chance, comme il était en fait un ami d'amis communs, j'ai eu l'occasion rare non pas de refaire l'entretien, ce qui ne marche jamais, mais d'installer un autre type 
d'entretien avec lui, deux ans plus tard, après un repas pris avec des amis chez lui. II m'a emmené, à ma demande, et à la suite de nos conversations à table sur l'opéra français, dans le salon de musique qu'il s'est aménagé, plus ou moins interdit à l'épouse, aux enfants et au chien. Et là, en situation, devant les objets et dans les lieux de sa passion, c'est un autre homme qui s'est livré, à un autre amateur. Non plus pour prouver à un sociologue qu'un interviewé n'est pas plus bête que lui, ef qu'en bon interactionniste, il peut en peu de temps lui donner ce qu'il attend et, en rajoutant sur sa sociologie, décliner complaisamment la série des déterminismes de son propre goût. Pas non plus pour commencer à réciter la liste plate de ses classiques favoris, tout en guettant mes réponses, selon un autre rituel, plus pauvre, celui par lequel les amateurs prennent souvent un premier contact. Mais pour montrer ce qu'il faisait, ses gestes, ses manies, ses "trucs", son installation. Et la gestion de ses moments de plaisir, le choix de ses disques, innombrables, les façons dont il s'approvisionnait, jusqu'à ses petites listes de critiques cochées (mais jamais suivies dans le magasin...), ou sa façon de caractériser ses humeurs et de traduire son état de fatigue en termes de répertoire possible.

Et il m'a montré les deux murs couverts de rayonnage de sa discothèque, remplis de disques, de $C D$ et de cassettes. C'est alors qu'en riant, il m'a expliqué son classement : "comme tout le monde", il avait commencé par la combinaison de chronologie et d'alphabet qui fait ressembler les rayons des particuliers à ceux d'une mini FNAC à domicile. Mais il est médecin, il est débordé, et il achète beaucoup. Il avait donc laissé des rayons vides, en bas à droite de son meuble, où empiler les achats récents, "à classer". Mais aussi à écouter en priorité, comme nouveautés. Et c'est là qu'il a eu l'idée de transformer en principe de rangement ce non-classement. Désormais, il met en bas à droite les enregistrements qu'il vient d'écouter, et il laisse peu à peu se déplacer ses disques à l'intérieur de sa bibliothèque, en fonction des faveurs plus ou moins récentes qu'ils ont reçues. D'abord instauré pour les coffrets d'opéra, son genre favori, ce système lui a tellement plu qu'il l'a généralisé, malgré les transferts périodiques d'étagères qu'il nécessite : mais, à un moment donné, précisément lorsqu'une perte de temps serait désagréable, le rangement est instantané. Et, surtout, sa discothèque se transforme peu à peu en une photographie sédimentée de ses goûts. L'espace physique de sa bibliothèque est devenu la trace de l'histoire personnelle de l'amateur.

II l'uilise comme telle, sachant qu'il va aller plutôt à droite pour écouter des choses nouvelles ou familières, mais qu'il veut répéter, plutôt en haut à gauche pour réécouter des choses plus rares, ou oubliées depuis longtemps. II me fait lui-même remarquer qu'il a trouvé le classement qui soit absolument personnel : qui d'autre que lui (et encore...) peut désormais savoir, dans l'autre sens, où est un disque précis? ! Mais le principal, c'est que l'amateur a triomphé du musicologue. C'est son goût qui préside au classement, non l'histoire de la musique... Entre l'entretien d'une mémoire amusée de ce qui s'est passé, la satisfaction de se tromper et de mesurer ses fausses impressions, le plaisir de se forcer à sortir de ses propres routines grâce à son dispositif, quand il doit se rabattre sur le disque à côté, il est facile de lire à sa jubilation qu'il n'a rien perdu à l'affaire! 


\section{Trio : le chanteur, le professeur et le sociologue...}

Au cours des expériences tentées dans le cadre du séminaire "Aimer la musique", pour définir et mettre en œuvre une sociologie pragmatique du goût ${ }^{8}$, une question de méthode très classique se pose de façon récurrente, portant sur l'engagement variable et réflexivement contrôlé du sociologue dans la pratique qu'il observe. La simplicité et le résultat spectaculaire d'une de ces expérimentations nous ont frappés. Elle portait sur des cours particuliers de chant, que l'une d'entre nous (musicienne, "sans plus"... est allée observer, carnet de notes et magnétophone à la main, sur une longue période. À y repenser, c'est moins l'« écoute» qui posait problème que, avant cela, quelque chose comme l'« entente" : le fait même de percevoir ce dont il était question entre le professeur et l'élève.

La consigne était restée la même, tout au long de l'expérience : " tout " noter. Nous défiant des effets de séduction et de plaisir, de projection et d'identification, et aussi de celui des faux savoirs sur le chant et la musique, favorisant les réinterprétations ex post, nous voulions pouvoir revenir sur les moindres impressions faites sur le tas sans trop savoir alors à quoi elles rimaient. Dans le détail, il reste beaucoup à tirer de ces notations. Mais pour le point qui nous occupe ici, elles suivent un chemin étonnamment rectiligne : au début, toutes portent sur les corps, les postures, les mimiques, les gestes, les mots clés incompréhensibles et les borborygmes, les raclements de gorge, les hésitations, les échanges de signes entre le professeur et l'élève. À la fin, il n'est plus question que de voix, de sonorité, d'effets sur l'auditeur, de qualité des voyelles, de beauté relative " c'est mieux", "c'est moins beau que juste avant »...., de sourires à tel passage, de succès ou d'échecs de telle reprise, avec un recours fréquent aux termes des acteurs : ouverture, couverture, fermeture, résonance, voile, etc. Si l'on prolonge l'histoire, elle reste tout aussi linéairement prévisible: la sociologue prend désormais elle-même des cours de chant.

Il y a un côté œuf de Christophe Colomb dans ce constat, qui en dit plus sur les a priori des sociologues que sur le chant. En simplifiant, on passe d'un compte rendu à $100 \%$ de social et $0 \%$ de musical à un rapport inverse. L'expérience a bien mis en évidence l'effet pervers d'une théorie sociologique qui transforme toutes les activités en prétextes indifférents de jeux dont le principe est social, parce qu'elle traduit en un problème de croyance un problème de pertinence. Ce qu'a peu à peu acquis l'observatrice, ce n'est pas une initiation, c'est une capacité à sentir ce qui compte pour les chanteurs. En insistant sur l'opposition entre l'enjeu d'une activité, auquel les participants sont exclusivement sensibles, et l'installation sociale de cet enjeu, ou il-lusio, pour reprendre la célèbre étymologie soulignée par Bourdieu, investissement arbitraire qu'au contraire les participants ne veulent et ne peuvent pas voir (le sociologue le révélant malgré leur résistance), la théorie du social comme croyance généralisée a fini par transmuer miraculeusement en compétence socio-
8. «Aimer la musique. Sociologie de la musique, histoire de l'amateur, musicologie du goût", séminaire de recherche CSIEHESS-CNRS, animé depuis 1997 par J.M. Fauquet, A. Hennion et G. Teil. Un compte rendu de diverses séances et une présentation de la démarche d'ensemble ont paru dans la Revue de musicologie (HENNION, 2002) et la revue MEI (HENNION, 2003a). Sur la pragmatique du goût, voir HENNION (2003b). 
logique l'ignorance technique, rebaptisée prise de distance et recul réflexif. Moins on s'y connait, moins on risque de se "faire avoir" par la croyance des acteurs.

Mais il n'existe rien de tel qu'un "objet» de la musique, si évident qu'il ne serait plus vu comme tel de l'intérieur, par les initiés, et si facile à isoler qu'il n'y ait plus à se préoccuper que du travail social qui l'entoure, pour les sociologues. II n'y a pas d'issue du côté de l'extériorité, précisément parce que l'objet n'est pas "la musique", un donné fixe, qu'on pourrait isoler de l'activité, mais ce qui surgit avec elle, à travers elle. La sensibilité à des différences de qualité n'est pas donnée au départ, elle s'acquiert avec le temps. L'observatrice "apprend" à entendre pendant les cours, en même temps que le chanteur apprend à chanter. Réciproquement, une fois qu'elle entend, elle n'accorde plus guère d'attention à ce qui la frappait exclusivement au début, quand toute cette gesticulation lui paraissait largement arbitraire - pour reprendre un mot clé de la sociologie critique : les codes, les postures, le "chiquée, ou, pour le dire plus franchement, le caractère un peu ridicule des attitudes, au contraire non perçu par le prof et l'élève.

La leçon est simple et radicale, si on la fait porter sur les sociologues lelle l'est moins pour l'observatrice, qui n'est pas sortie d'affaire en ce qui concerne le chant...) : tant qu'on n'est pas sensible à ce qui compte pour les participants, on fait de la sociologie. On déchiffre des codes, on repère des rituels, on relève des interactions. On voit des indigènes s'agiter avec leurs gris-gris. Si au contraire on s'engage, dans le temps et avec son corps, dans le commerce avec l'objet en cause, c'est ce rapport qui prend le dessus, et rend bientôt frivoles ou "sans objet» les éléments relevés par l'observateur non initié. L'écoute est bien aussi un défi à la sociologie : que peux-tu dire de la musique, sans mettre en jeu ton propre amour de la musique, et d'abord, sans écouter toi-même? le mot "écoute" est parfait, pour cela, il évite la dualité du rapport à l'objet ls'y connaître ou non, l'aimer ou non, se "faire avoir" en y croyant ou montrer la croyance en l'objett, pour rameuter une large palette d'aspects variés de l'activité musicale : I'attention d'un "je», la présenceréférence à d'autres, l'appareillage de l'oreille, les instruments de la production sonore, la réaction corporelle et le développement réflexif d'une sensibilité.

\section{Odile, musicienne : mais est-ce que i'écoute de la musique, moi ?...}

Tu sais, en fait moi, paradoxalement, enfin paradoxalement ou non, je ne sais pas, mais je n'écoute pas tellement de musique, enfin comme ça, pour ça. Je te dis ça comme ça parce qu'on en a discuté ensemble, justement, avec des potes musicos aussi. J'en joue, je répète, je joue pour moi, i'en joue tout le temps, mais je n'ai pas vraiment le temps d'aller acheter des disques et d'en mettre, pour moi. Le temps ni l'envie, peut- 
être, c'est de ça qu'on discutait, est-ce vraiment un mal e j'écoute professionnellement, quand on joue un truc, oui on me passe le disque et i'écoute ce qu'ils ont fait, et puis au contraire la radio, comme ça, sans faire attention, tout ce qu'on me dit que ce n'est pas bien, en faisant la cuisine ou dans la bagnole, pas forcément du classique ou du contemporain d'ailleurs, mais je ne suis pas du tout collectionneuse. C'est ça, pour moi, un amateur, c'est un collectionneur d'enregistrements, pas quelqu'un qui "vit" de la musique, aux deux sens du terme, comme moi.

[plus loin] II faut être honnête, je me moquais un peu des amateurs mais dans l'autre sens, des fois je me dis que je m'y connais bien moins que d'autres, que des gens qui ont plein de disques, et qui connaissent tout, des versions, des compositeurs dont je ne sais même pas le nom. Je ne sais pas, c'est deux choses différentes, quoi.

La variété et la mutation continue de l'expérience musicale que cette instrumentiste professionnelle s'étonne tout haut de ne pas trouver dans l'écoute comme le font ses amis amateurs, elle l'obtient sans doute dans le jeu. Mais elle va nous servir à conclure sur la répétition. Car c'est à une invention majeure, l'écoute répétée, rendue possible par l' "enregistrement», que renvoient en définitive nombre de ces tactiques d'écoute dont parlent les amateurs, et que mentionne explicitement l'un d'eux : choisir, revenir, repasser, répéter, c'est faire apparaître une musique, une œuvre ou telle version, un court passage ou au contraire l'ensemble d'un opéra, sous de multiples facettes, au fur et à mesure qu'on en connaît tous les tours et les ressorts et qu'elle peut en livrer d'autres, tandis qu'en face d'elle varient aussi les états et dispositions d'un écouteur dont l'attente et l'attention changent au fil de ces répétitions. C'est la variété des attentions possibles, justement, qui se trouve multipliée à l'infini, loin de la focalisation sur une logique intrinsèque de l'œuvre, dont l'écoute ne serait que la servante.

\section{Conclusion : à la base de la définition moderne de la musique, la répétition}

Cette répétition relayée par l'enregistrement entraîne à la fois vers des "plus", une surattention à des détails ou des aspects que l'unicité, la position fixe et la temporalité contraignante du concert interdisent - je pense ici à un autre collectionneur, amateur d'opéras peu courus et grand connaisseur du chant français de l'entre-deuxguerres, qui s'est peu à peu constitué un ensemble unique d'enregistrements, sur tous les supports disponibles, qu'il connaît très bien; il a, à juste titre, tout à fait conscience qu'il dispose d'une compétence et d'une sensibilité aux voix et aux nuances les plus infimes de l'enregistrement, que personne n'a été en position de maîtriser avant lui - et que seul le disque a pu lui permettre de forger. 
Elle entraîne aussi des "moins", au sens de l'esthétique ou de la musicologie : les techniques d'enregistrement donnent désormais tous les moyens de mettre l'œuvre à distance, ou de l'instrumentaliser comme fond sonore, comme support de ses rêves ou de sa gymnastique, de la laisser se dérouler sans y prêter attention, ou encore, loin d'en faire proliférer la variété et d'en multiplier les potentiels, de l'user dans une insistance obsessionnelle, en la répétant à satiété, comme pour lui faire rendre l'âme... Autant dire qu'il peut très bien s'agir aussi de "plus", à condition de remettre l'usager de la musique au cœur de l'analyse!

Mais j'ai conscience que, renonçant prudemment à esquisser la double réécriture de la musique comme histoire et de la musique comme présent à laquelle, en insistant sur l'invention de l'écoute comme disposition, invitait mon ambitieuse introduction, c'est à un exercice plus modeste que je me suis livré, à partir des enquêtes conduites auprès de ces amateurs ${ }^{9}$. Pour autant, je crois que, en donnant la parole à des amateurs (et ce "donner » peut-être entendu au sens fort), il a permis de repérer divers éléments récurrents, que l'insolente question posée, "qui écoute? 》, avait fait ressurgir, qu'elle laissait déborder de la musique. Éléments dont je résume ici la liste, à titre de conclusion provisoire :

- le corps qui entre en contact;

- l'organisation d'un moment d'attention;

- les appuis et les techniques collectivement produits pour rendre l'écoute possible;

- et enfin la définition même de l'objet en cause - un enjeu qui soit «à venir», de la musique à faire advenir, et non déjà là. État incertain, effet à retrouver, exploration d'un monde commun, transport entre des points inconnus: l'écoute est une façon de faire apparaitre la musique, de la faire surgir en soi, et non de la recevoir comme une hostie.

\section{Bibliographie}

ADORNO, T. W. (1994), Introduction à la sociologie de la musique. Douze conférences théoriques, Orgemont/Genève, Contrechamps.

BESSY, C. et F. CHATEAURAYNAUD (1995), Experts et faussaires, Paris, Métailié.

BOURDIEU, P. (1979), La distinction. Critique sociale du jugement, Paris, Minuit.

DE CERTEAU, M., L. GIARD, et al. (1980), L'invention du quotidien, Paris, UGE.

DeNORA, T. (2000), Music in Everyday Life, Cambridge, Cambridge University Press.

HENNION, A. (1981), Les professionnels du disque. Une sociologie des variétés, Paris, A.M. Métailié.

HENNION, A. et S. MAISONNEUVE (2000), Figures de l'amateur. Formes et pratiques de l'amour de la musique aujourd'hui, Paris, La Documentation française.
9. Sans prétendre qu'ils aient rempli un tel programme, c'est plutôt pour m'excuser de ne pas avoir repris ici des éléments d'analyse déjà esquissés que je me permets de renvoyer à ces travaux au long cours, cf. supra, $n^{\circ} 1$. 
HENNION, A. (2002), « L'écoute à la question», Revue de musicologie, vol. 88, n 1, p. 95-149.

HENNION, A. (2003a), "Une pragmatique de la musique : expériences d'écoutes", MEI 17, "Musique. Interpréter l'écoute", p. 31-43.

HENNION, A. (2003b), "Ce que ne disent pas les chiffres... Vers une pragmatique de l'amateur", dans DONNAT, O. (s. la dir. del, Le(s) public(s). Politiques publiques ef équipements culturels, Paris, Presses de la FNSP.

MAISONNEUVE, S., G. TEIL, et al. (2002), Le goût comme un "faire ensemble», Paris, CSIMission du Patrimoine/Ministère de la culture.

SZENDY, P. (2001), Écoute. Une histoire de nos oreilles, Paris, Minuit.

TEIL, G. et A. HENNION (2003), Les protocoles du goût. Une pragmatique de l'amateur, Paris, CSI-DEP/Ministère de la culture.

WEBER, W. (1997), "Did People Listen in the 18 $8^{\text {th }}$ Century? ", Early music, p. 678-691. 


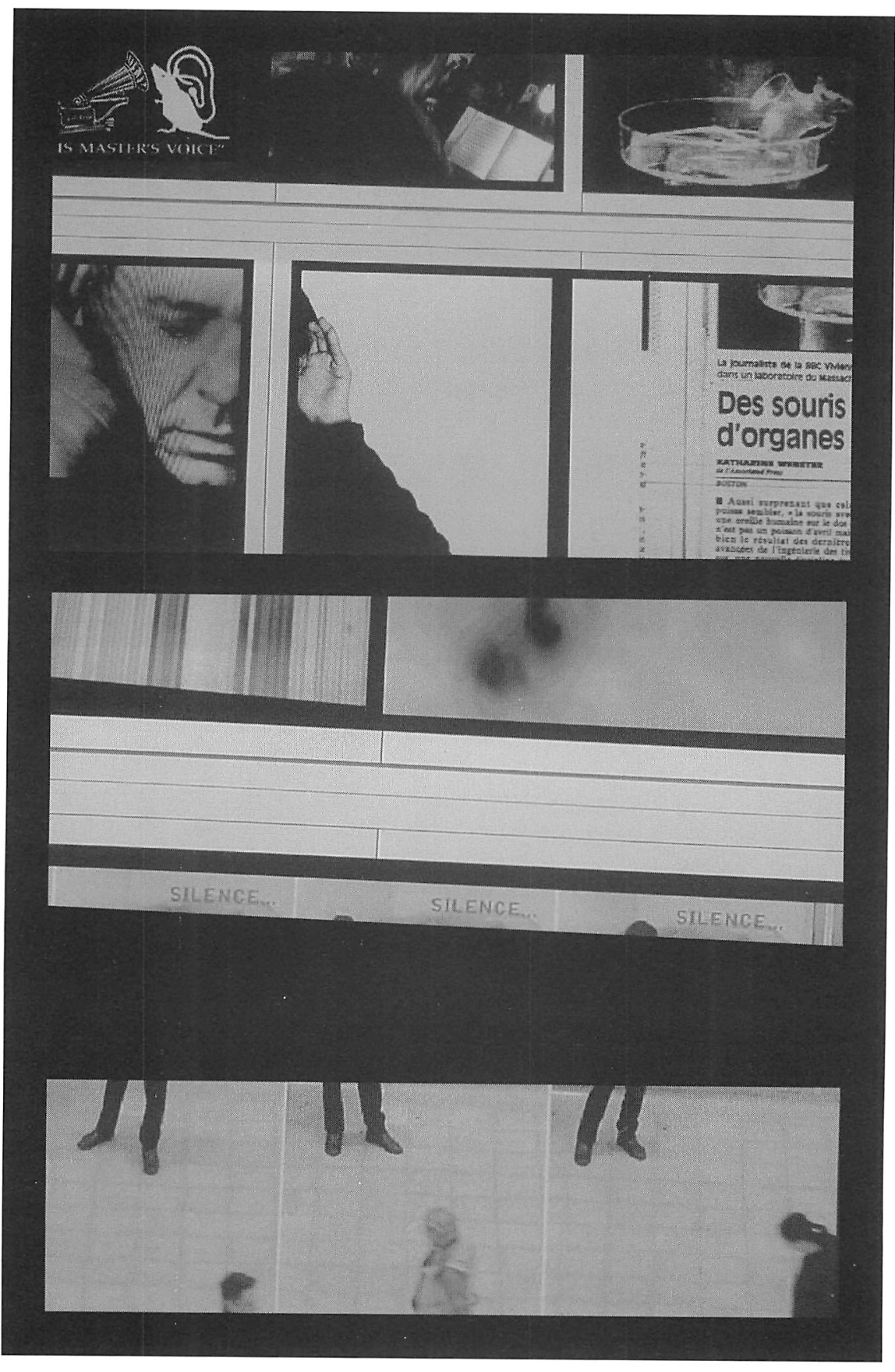

